\title{
How Simultaneous Using of Photo Biomodulation and Natural Killer Cells is Effective in Treatment of Cancer?
} \author{
Lazar $^{5}$ and Mehdi Shamsara ${ }^{3 *}$ \\ ${ }^{1}$ Department of Biology, Sistan and Baluchestan University. Iran \\ ${ }^{2}$ Middle East-Gene therapy Company, Iran \\ ${ }^{3}$ Department of Animal Biotechnology, National Institute for Genetic Engineering and Biotechnology, Iran \\ ${ }^{4}$ Department of Plant Biotechnology, National Institute for Genetic Engineering and Biotechnology, Iran \\ ${ }^{5}$ NIKASSA Biophotonics corp., Iran
}

Najmeh Malekzadeh Gonabadi ${ }^{1}$, Sahar shojaee ${ }^{2}$, Elmira Vanaki ${ }^{3}$, Seyed Mehdi Alavi ${ }^{4}$, Albert

*Corresponding author: Mehdi Shamsara, Department of Animal Biotechnology, National Institute for Genetic Engineering and Biotechnology, Tehran, Iran

To Cite This Article: Najmeh Malekzadeh Gonabadi, Sahar shojaee, Elmira Vanaki, Seyed Mehdi Alavi, Albert Lazar, Mehdi Shamsara. How Simultaneous Using of Photo Biomodulation and Natural Killer Cells is Effective in Treatment of Cancer?. Am J Biomed Sci \& Res. 2021 - 13(2). AJBSR.MS.ID.001850. DOI: 10.34297/AJBSR.2021.13.001850.

Received: 啙 May 24, 2021; Published: 眥 June 14, 2021

\begin{abstract}
There are many types of cancer treatments. The types of treatment that you receive will depend on the type of cancer you have and how advanced it is. In this article, the mechanism of low-level laser in killing cancer cells and how this treatment effects on the patient's immune cells; and natural killer cells injected in immunotherapy are investigated to evaluate the simultaneous effect of these two treatments. Our opinion is that despite the effect of PBM on growth and proliferation, melatonin is produced through the activation of NF-kB through the mitochondrial pathway, which is produced more rapidly by suppressing NF-kB and leads to apoptosis of cancer cells. On the other hand, the effect of melatonin on injected NK cells makes the treatment more effective.
\end{abstract}

Keywords: Cancer; Immune cell therapy; Natural killer cell; Photo biomodulation

\section{Expert Opinion}

PBM affects the thymus, liver and stem cells in these areas, causing the production and proliferation of more stem cells [1]. PBM may also cause more injected NK cells to proliferate and more NK cells to penetrate the tumor environment. Another hypothesis is that PBM prepares the tumor environment for more efficient NK cell function. In the ion channel pathway, because ion channels are photoreceptors, the effect of PBM on membranes and ion channels, in addition to the effect on cell apoptosis, which prevents tumor growth, may lead to greater uptake of NK cells by cancerous cells and increase tumor permeability to immune cells, including injected NK cells [2-4]. In Melatonin and PI3K pathways, because melatonin suppresses the PI3K pathway, it prevents cell proliferation and induces cell apoptosis. Because the PI3K pathway requires many receptors to be activated, another hypothesis is that PBM on channels, alpha protein helix, inhibitory effect on PI3k amplitude catalyst may not be maintained by receptor adaptation change, and signaling cascade may not be initiated $[1,5]$. It has been hypothesized that the rapid production of melatonin is due to the faster uptake of ATP in damaged cells. In addition, if melatonin production by this pathway occurs faster than activation of the tyrosine kinase receptor pathway, melatonin suppresses NF-KB, while lasers activate NF-KB from other pathways, eventually 
leading to apoptosis of cancer cells. Therefore, it is assumed that because the type of malignant cell is different from the benign cell, the type and level of agents will be different. Therefore, the uptake of PBM by the cell and the induction pathways will also be different.

\section{PBM and NK Cells, a Synergistic Combination}

NK treatment with cytokines such as IL- 2 or with histamine has a better therapeutic effect. Here PBM eventually produces stimulatory cytokines from different pathways. On the other hand, the production of melatonin with an inhibitory effect on inhibitory factors contributes to the better effect of NK cells. The IL-2 produced by PBM will increase the number of NK cells. In fact, PBM has a therapeutic effect due to a combination of different factors. Another hypothesis in this combination therapy is the effect of PBM on cell membranes and receptors [6]. Therefore, it is hypothesized that this change in calcium concentration and membrane permeability may affect the permeability, function, as well as the production of more NK cells than primary stem cells Therefore, PBM will be introduced as a therapeutic combination with NK because it increases cell proliferation through the stem cell pathway. On the other hand, it activates NK stimulants such as cytokines and suppresses inhibitory factors through various pathways. In addition, the effect of the laser on the cell alone causes apoptosis and prevents cell proliferation. Some of the factors produced, especially melatonin with rapid production in mitochondria, help increase the proliferation of natural killer cells and apoptosis of cancer cells by acting in different ways. Another hypothesis in this combination therapy is the effect of PBM on cell membranes and receptors. PBM can increase the availability of NK cell activating receptors to make NK cells perform better. In PBM studies contradictory results have been reported. It is hoped that by completing the effective laser protocol and more accurate results, we will achieve more effective treatment combinations for cancer.

\section{Conflict of Interest Statement}

The authors state no conflicts of interest regarding this study.

\section{References}

1. Hamblin MR (2018) Mechanisms and Mitochondrial Redox Signaling in Photobiomodulation. Photochem Photobiol 94(2): 199-212.

2. Salari V, Barzanjeh S, Cifra M, Simon C, Scholkmann F, et al. (2018) Electromagnetic fi elds and optomechanics in cancer diagnostics and treatment. Front Biosci (Landmark Ed) 23: 1391-1406.

3. Kim HP (2014) Lightening up Light Therapy: Activation of Retrograde Signaling Pathway by Photobiomodulation. Biomol Ther (Seoul) 22(6): 491-496.

4. Calabro E, Magazu S (2018) Resonant interaction between electromagnetic fields and proteins: A possible starting point for the treatment of cancer. Electromagn Biol Med 37(3): 155-168.

5. Liebert AD, Bicknell BT, Adams RD (2014) Protein conformational modulation by photons: a mechanism for laser treatment effects. Med Hypotheses 82(3): 275-281.

6. Mortezaee K, Potes Y, Mirtavoos-Mahyari H, Motevaseli E, Shabeeb D, et al. (2019) Boosting immune system against cancer by melatonin: A mechanistic viewpoint. Life Sci 238: 116960. 